\title{
Erratum: Low-energy effective theory in the bulk for transport in a topological phase [Phys. Rev. B 91, 125303 (2015)]
}

Barry Bradlyn and N. Read

(Received 31 May 2016; published 13 June 2016)

DOI: 10.1103/PhysRevB.93.239902

The following sentence should be added to the acknowledgments: "The authors are grateful for support from NSF Grant No. DMR-1408916 while the paper was revised". 